\title{
Schwannoma extending from the umbilical region to the mid-thigh, compressing the major vessels of the right leg: A case report and review of the literature
}

\author{
J Yorke, ${ }^{1,2}$ MD; B M Duduyemi, ${ }^{3}$ FMCPath; A C Yifieyeh, ${ }^{2}$ FWACS; P K S Fiifi-Yankson, ${ }^{2}$ MB ChB; C Appiah, ${ }^{2}$ MB ChB; \\ D Afful-Yorke, ${ }^{2}$ BSc; M O Adinku, ${ }^{2}$ FWACS; D Ahulu, ${ }^{2}$ BSc \\ ${ }^{1}$ Department of Surgery, School of Medical Sciences, College of Health Sciences, Kwame Nkrumah University of Science and Technology, \\ Kumasi, Ghana \\ ${ }^{2}$ Directorate of Surgery, Komfo Anokye Teaching Hospital, Kumasi, Ghana \\ ${ }^{3}$ Department of Pathology, Kwame Nkrumah University of Science and Technology, Kumasi, Ghana
}

Corresponding author: B M Duduyemi (babsdudu@yahoo.com)

\begin{abstract}
Schwannomas are benign, usually encapsulated, nerve sheath tumours derived from Schwann cells. They commonly arise from the cranial nerves as acoustic schwannomas and are extremely rare in the pelvis and retroperitoneal area $(<0.5 \%$ of reported cases) unless they are combined with Von Recklinghausen disease (type 1 neurofibromatosis). We report the case of a 23-year-old woman with a mass extending from the umbilical region in the abdomen to the upper two-thirds of the thigh. As this tumour is so rare, and in order to ensure optimal treatment and survival for our patient, a computed tomography-guided biopsy was performed before en bloc tumour excision. Because of the possibility of malignancy, complete excision of the mass was performed, with pelvic blunt dissection. Histological examination showed a benign neoplasm, originating from the cells of peripheral nerve sheaths; the diagnosis was a schwannoma. Abdominal schwannomas are rare neoplasms that can be misdiagnosed. Laparoscopy is a safe and efficient option for approaching benign pelvic tumours and may offer the advantage of better visualisation of structures owing to the magnification in laparoscopic view, especially in narrow anatomical spaces. However, in our case laparoscopy was not considered owing to the size and anatomical location of the tumour.
\end{abstract}

S Afr Med J 2016;106(7):692-694. DOI:10.7196/SAMJ.2016.v106i7.10252

Schwannomas are benign, usually encapsulated, nerve sheath tumours derived from Schwann cells. One of the most common types of benign peripheral nerve sheath tumours, they are common in cranial and peripheral nerves but are rarely located in the pelvis. ${ }^{[1,2]}$ Schwannomas can occur sporadically or as manifestations of genetic conditions such as neurofibromatosis types 1 and 2. Pelvic schwannomas have no specific radiological features and are often considered to be urological diseases or gynaecological masses. ${ }^{[3,4]}$ We report the rare case of a woman with a pelvic schwannoma in the right parametrium and extending into the right thigh.

\section{Case report}

A 23-year-old woman was referred to a general surgery outpatient department for management of an abdominal mass of increasing size and sustained pain in the right leg that had been present for 13 years. The pain was independent of activity, and the patient had no sign of neurovascular deficit. The swelling had progressively increased in size since it was first noticed and was initially associated with occasional dull intermittent pain that radiated to the right lower limb.
Five months before presentation, the mass became extremely painful. The pain was throbbing and stabbing in nature, constant, radiated to the right lower limb, and was exacerbated by movement and relieved by rest. There was associated swelling of the right lower limb on standing for long periods, numbness and paraesthesiae. There was no history of other gastrointestinal or cardiovascular symptoms. The patient denied any unexplained weight loss and had a negative history for cancer. Her medical, surgical, obstetric and family history was unremarkable.

Physical examination revealed a wellnourished young woman, afebrile, anicteric, not pale and well hydrated, with a dumbbellshaped mass on the right lumbar region of the abdomen extending to the right thigh and measuring $25 \times 20 \mathrm{~cm}$ (Fig. 1). The mass was smooth and firm with well-defined edges and tender around the femoral region, and was attached to the underlying structures but not to the skin. There were varicosities over the mass at the femoral region but no lymphadenopathy. There was full power in all the muscles, and sensation was normal in the right lower limb. The femoral, popliteal, posterior tibialis and dorsalis pedis pulses

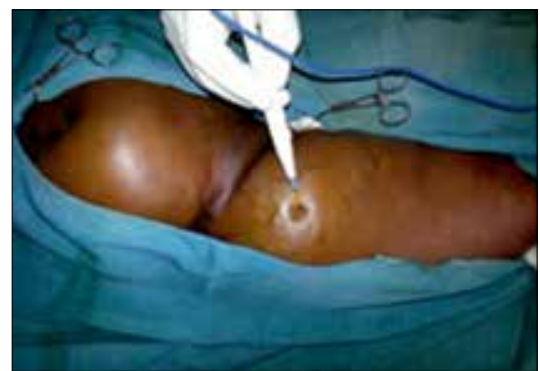

Fig. 1. Firm mass extending from the region of the umbilicus to the upper two-thirds of the right thigh.

were all palpable with adequate capillary refill in all the digits.

The results of routine haematological investigations and urinalysis as well as testing for several tumour markers, including carcinoembryonic antigen, $a$-fetoprotein and carbohydrate antigen 19.9, were all within normal limits.

\section{Imaging}

Imaging studies included plain radiographs, ultrasonography and a computed tomography (CT) scan of the abdomen and thigh.

Radiographs of the right hip and thigh revealed soft-tissue swelling with no bone deformities. A chest radiograph was normal. 


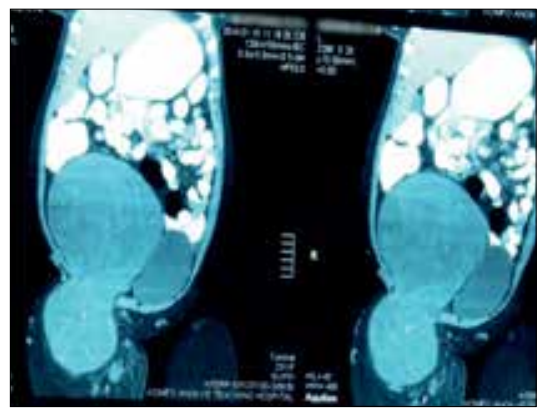

Fig. 2. Axial contrast-enhanced CT image of the abdomen and upper thigh, showing the dumbbell-shaped mass.

Serial axial contrast-enhanced CT images showed a $26.5 \times 26.3 \times 16.3 \mathrm{~cm}$ welldefined dumbbell-shaped heterogeneously enhancing mass arising at the level of L4 on the right and extending inferiorly lateral to the right femoral vessels to the anterior compartment of the proximal right femur, about $7 \mathrm{~cm}$ inferior to the pubic symphysis. The mass had internal calcifications and internal areas of hypodensity suggestive of cystic degeneration. It also exerted a mass effect, displacing bowel loops superiorly, and compressing and displacing the urinary bladder, uterus, distal inferior vena cava and right iliac and femoral vessels to the contralateral side.

The mass was in close proximity to the femoral vessels, but was not seen to infiltrate or spread to adjacent organs. The bones appeared normal, with no remodelling or lytic or sclerotic lesion seen (Fig. 2).

\section{Diagnosis and treatment}

A diagnosis of right femoral nerve schwannoma was made. Total removal of the mass (Fig. 3) was achieved through laparotomy and extension of the incision to the upper thigh.

Intraoperative findings were a right retroperitoneal mass extending from below the right kidney to the upper two-thirds of the right thigh with superficial varicosities. The right kidney, ovary, ureter, femoral artery and vein, liver and bladder were normal.

The preoperative findings indicated that we had to deal with a retroperitoneal tumour of unknown pathology in a nulliparous woman in her reproductive years. The abdomen was opened through an extended lower midline incision. A large retroperitoneal mass, measuring $26.5 \times 26.3 \times$ $16.3 \mathrm{~cm}$, was noted in the right parametrium and in close proximity to the internal iliac vessels. Complete excision of the mass was performed. Histological examination showed a benign neoplasm originating from the cells of the peripheral nerve
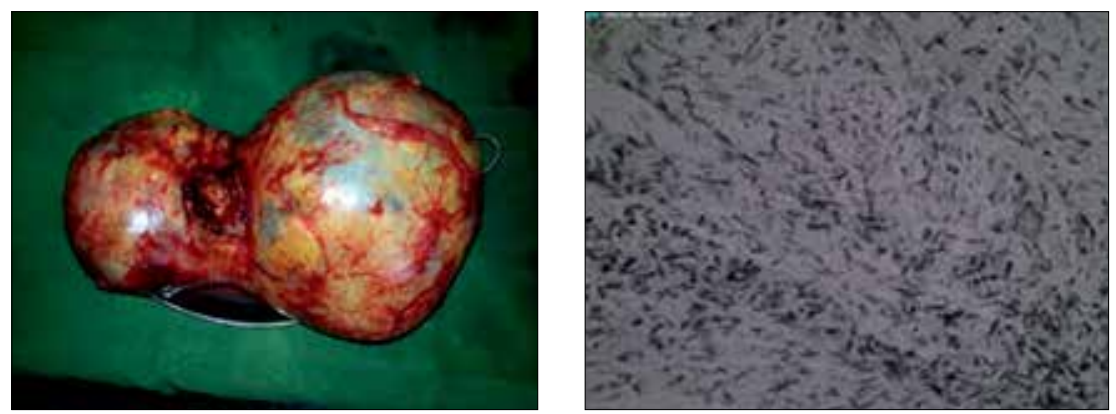

Fig. 3. Surgically resected well-defined and encapsulated dumbbell-shaped mass measuring $26.5 \times 26.3 \times 16.3 \mathrm{~cm}$.

sheath consistent with a schwannoma (Fig. 4). The patient recovered well, was discharged on postoperative day 10 and was seen again for follow-up 2 months after surgery (Fig. 5).

\section{Discussion}

Schwannomas are nerve sheath tumours that arise in peripheral, cranial or visceral nerves at any anatomical site in the human body. ${ }^{[5,6]}$ These tumours commonly arise from the cranial nerves as acoustic neurinomas, but they are extremely rare in the pelvis and the retroperitoneal area $(<0.5 \%$ of reported cases), unless they are combined with Von Recklinghausen disease (type 1 neurofibromatosis). ${ }^{[6]}$ Schwannomas are non-aggressive, slow-growing, solitary neoplasms with an extremely low possibility of malignant transformation or recurrence after excision. ${ }^{[7]}$ Macroscopically, schwannomas are solitary, well-circumscribed and encapsulated tumours. ${ }^{[4]}$

As a result of their slow growth rate and anatomical location, pelvic schwannomas remain asymptomatic and are discovered either incidentally during a medical investigation for unrelated symptoms or when they become sufficiently large to cause a mass effect. ${ }^{[7,9]}$ This mass effect can lead to pain in the pelvic area and lower back and a sense of heaviness accompanied with urinary and digestive symptoms caused by bladder and bowel compression. ${ }^{[9]}$ Pelvic schwannomas are not easily diagnosed. The clinical signs and symptoms are not pathognomonic for this pathological entity.

Our case encouraged us to conduct a literature review and document cases of abdominopelvic schwannomas. The small number of these cases indicates the rarity of retroperitoneal abdominopelvic schwannomas. One case was a retroperitoneal pelvic schwannoma located in the right paracolpium, ${ }^{[10]}$ and another was a schwannoma present at the L5 vertebral body and causing pelvic pain. ${ }^{[1]}$ Takeuchi et al. ${ }^{[12]}$
Fig. 4. Histological section of a benign spindle cell neoplasm with admixture of cellular areas (Antoni A) and loosely cellular areas (Antoni B) consistent with a schwannoma.

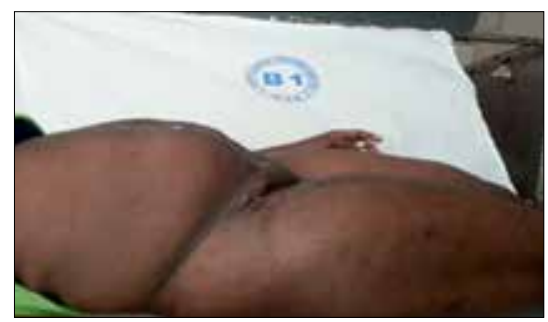

Fig. 5. The patient at follow-up 2 months after surgery.

reported 11 presacral and lateral pelvic region neurilemomas, 8 of which were located in the right or left presacral area and 3 in the lateral extraperitoneal region. One pelvic schwannoma of the anterior surface of the sacrum mimicking an ovarian cyst was also reported. ${ }^{[13]}$ An additional case of a retroperitoneal schwannoma localised in the pelvic cavity with complete cystic degeneration, mimicking an ovarian carcinoma, was reported, ${ }^{[14]}$ and there was one report of a presacral neurilemoma. ${ }^{[15]}$ One pelvic retroperitoneal neurilemoma arose in the fallopian tube, ${ }^{[16]}$ and one malignant schwannoma arose in the cervix. ${ }^{[17]}$ Yadav et al. ${ }^{[18]}$ reported one case of neurilemoma located in the right adnexa, and Sinha et al. ${ }^{[9]}$ reported two cases of pelvic schwannomas located in the broad ligament, with the clinical expression of a broad-ligament myoma. One reported case of neurilemoma of the pelvis mimicked a myoma of the uterus. ${ }^{[19]}$ Pelvic tumours, and specifically pelvic schwannomas, can cause chronic pelvic pain, as was reported in a case of a femoral nerve schwannoma with the clinical expression of chronic pelvic pain. ${ }^{[6]}$ There were two cases of obturator nerve tumours arising from Schwann cells, one expressed as a pelvic tumour and the other as an ovarian tumour. ${ }^{[20]}$ Nine cases of schwannomas mimicking ovarian malignancies have also been reported. ${ }^{[21,22]}$

Pelvic schwannomas, as mentioned above, are easily misdiagnosed owing to the lack of 
specific symptoms. Treatment is complete excision of the tumour, either laparoscopically or with open abdominal surgery. ${ }^{[23]}$ Because the vast majority of schwannomas are benign tumours, simple tumour enucleation could also be effective. In these surgical approaches it is important that the surgery does not result in neural lesions. ${ }^{[7]}$ The presacral-retrorectal space is considered to be a downward extension of the retroperitoneal space because it communicates superiorly with the latter at the level of peritoneal reflection (S2 - S3 vertebrae); surgical extirpation of presacral and pelvic tumours may therefore present several difficulties owing to limited access and poor visualisation in a narrow pelvis. ${ }^{[23,24]}$

Schwannomas are solitary, well-circumscribed, encapsulated tumours, and do not invade local tissues. ${ }^{[4]}$ Owing to these characteristics they are easily dissected from adjacent tissues, which makes laparoscopic resection possible. Laparoscopy is a safe and efficient option for approaching benign pelvic tumours and may offer the advantage of better visualisation of structures because of the magnification of the laparoscopic view, especially in narrow anatomical spaces. ${ }^{[23]}$ Our literature review indicated that laparoscopic excision of these neural tumours is the treatment of choice. We used open surgery in our case because of the large size of the tumour and the extension into the thigh.

Of note is that many pelvic schwannomas were misdiagnosed, and were discovered during an operation that was considered to be the optimal therapy for the initial diagnosis.

Acknowledgment. The patient provided written consent for this case report.

1. Rao W, Wang G, Xiu D. Laparoscopic resection of a retroperitoneal schwannoma adherent to vital vessels. Surg Laparosc Endosc Percutan Tech 2009;19(1):e21-e23. DOI:10.1097/SLE.0b013e3182365a3f 2. Funamizu N, Sasaki A, Matsumoto T, Inomata M, Shiraishi N, Kitano S. Laparoscopic resection of a retroperitoneal schwannoma behind the lesser omental sac. Surg Laparosc Endosc Percutan Tech 2004;14(3):175-177. DOI:10.1097/01.sle.0000129379.96203.66
3. Mehta M, Thurston WA, Merchant N, Murphy KJ. Obturator nerve schwannoma presenting as an adnexal mass: Case report. Can Assoc Radiol J 1999;50(1):20-22.

4. Daneshmand S, Youssefzadeh D, Chamie K, et al. Benign retroperitoneal schwannoma: A case series 4. Daneshmand S, Youssefzadeh D, Chamie K, et al. Benign retroperitoneal schwannoma: A case
and review of the literature. Urology 2003;62(6):993-997. DOI:10.1016/S0090-4295(03)00792-1

Borghese $\mathrm{M}$, Corigliano N, Gabriele R, et al. Benign schwannoma of the pelvic retroperitoneum: Rorghese M, Corigliano N, Gabriele R, et al. Benign schwannoma of a case and review of the literature. G Chir 2000;21(5):232-238.
Report of

Report of a case and review of the literature. G Chir 2000;21(5):232-238.
6. Dawley B. A retroperitoneal femoral nerve schwannoma as a cause of chronic pelvic pain. J Minim 6. Dawley B. A retroperitoneal femoral nerve schwannoma as a cause of
Invasive Gynecol 2008;15(4):491-493. DOI:10.1016/.j.jig. 2008.02 .007

Invasive Gynecol 2008;15(4):491-493. DOI:10.1016/j.jmig.2008.02.007
7. Strauss DC, Qureshi YA, Hayes AJ, Thomas JM. Management of benign retroperitoneal schwannomas 7. Strauss DC, Qureshi YA, Hayes AJ, Thomas JM. Management of benign retroperitoneal schwannon
A single-centre experience. Am J Surg 2011;202(2):194-198. DOI:10.1016/j.amjsurg.2010.06.036

8. White W, Shiu MH, Rosenblum MK, Erlandson RA, Woodruff JM. Cellular schwannom A clinicopathologic study of 57 patients and 58 tumours. Cancer 1990;66(6):1266-1275. DOI:10.1002/1097-0142(19900915)66:6\%3C1266:AID-CNCR2820660628\%3E3.0.CO;2-E

9. Sinha R, Sundaram M, Hegde A, Mahajan C. Pelvic schwannoma masquerading as broad ligament myoma. J Minim Invasive Gynecol 2008;15(2):217-219. DOI:10.1016/.jmig.2007.09.009

10. Ueda M, Okamoto Y, Ueki M. A pelvic retroperitoneal schwannoma arising in the right paracolpium. Gynecol Oncol 1996;60(3):480-483. DOI:10.1016/0020-7292(96)81558-X

11. Shoher A, Arbab F, Lucci A, Jr. Giant pelvic schwannoma with ancient change. J Am Coll Surg 2003;197(1):163. DOI:10.1016/S1072-7515(03)00339-9

12. Takeuchi M, Matsuzaki K, Nishitani H, Uehara H. Ancient schwannoma of the female pelvis. Abdom Imaging 2008;33(2):247-252. DOI:10.1007/s00261-007-9228-y

13. Ibraheim M, Ikomi A, Khan F. A pelvic retroperitoneal schwannoma mimicking an ovarian dermoid cyst in pregnancy. J Obstet Gynaecol 2005;25(6):620-621. DOI:10.1080/01443610500243752

14. Aran T, Guven S, Gocer S, Ersoz S, Bozkaya H. Large retroperitoneal schwannoma mimicking ovaria carcinoma: Case report and literature review. Eur J Gynaecol Oncol 2009;30(4):446-448.

15. Song JY, Kim SY, Park EG, et al. Schwannoma in the retroperitoneum. J Obstet Gynaecol Res 2007;33(3):371-375. DOI:10.1111/j.1447-0756.2007.00539.x

16. Duran B, Guvenal T, Yildiz E, Cetin M, Erden O, Demirkoprulu N. An unusual cause of adnexal mas: Fallopian tube schwannoma. Gynecol Oncol 2004;92(1):343-346. DOI:10.1016/j.ygyno.2003.09.032

7. Lallas TA, Mehaffey PC, Lager DJ, Van Voorhis BJ, Sorosky II. Malignant cervical schwannoma: A unusual pelvic tumour. Gynecol Oncol 1999;72(2):238-242.

18. Yadav Y, Onon T, Sukumar S. Conservative management of a pelvic Schwannoma presenting as an adnexal mass. J Obstet Gynaecol 2008;28(3):364-365. DOI:10.1080/01443610802066265

19. Chen $\mathrm{CH}$, Jeng CJ, Liu WM, Shen J. Retroperitoneal schwannoma mimicking uterine myoma. Taiwan J Cbstet Gynecol 2009;48(2):176-177. DOI:10.1016/S1028-4559(09)60282-5

20. Ningshu L, Min Y, Xieqiao Y, Yuanqing Y, Xiaoqiang M, Rubing L. Laparoscopic management of Ningshu L, Min Y, Xieqiao Y, Yuanqing Y, Xiaoqiang M, Rubing L. Laparoscopic management of
obturator nerve schwannomas: Experiences with 6 cases and review of the literature. Surg Laparosc obturator nerve schwannomas: Experiences with 6 cases and review of the litera
Endosc Percutan Tech 2012;22(2):143-147. DOI:10.1097/SLE.0b013e3182478870 21. Ozat M, Altinkaya SO, Gungor T, et al. Extraovarian conditions mimicking ovarian cancer: A single center
experience of 15 years. Arch Gynecol Obstet 2011;284(3):713-719. DOI:10.1007/s00404-010-1705-9

22. Korkontzelos I, Tsimoyiannis E, Zagaliki A, Demou A, Karabina E, Antoniou N. Pelvic retroperitonea schwannoma presenting as a gynecologic mass: Case report. Eur J Gynaecol Oncol 2005;26(1):117 119.

23. Konstantinidis K, Theodoropoulos GE, Sambalis G, et al. Laparoscopic resection of presacral schwannomas. Surg Laparosc Endosc Percutan Tech 2005;15(5):302-304. DOI:10.1097/01. sle. 0000183252.96808 .78

24. Wolpert A, Beer-Gabel M, Lifschitz O, Zbar AP. The management of presacral masses in the adult. Tech Coloproctol 2002;6(1):43-49. DOI:10.1007/s101510200008

Accepted 2 November 2015 